\title{
Amphotericin B Assembles into Seven-Molecule Ion Channels: An NMR and Molecular Dynamics Study
}

Yuichi Umegawa, ${ }^{1,2 *}$, Tomoya Yamamoto ${ }^{1 * \dagger}+$, Mayank Dixit ${ }^{3 *}$, Kosuke Funahashi $^{3}$, Sangjae Seo $^{3}$, Yasuo Nakagawa $^{1}$, Taiga Suzuki ${ }^{1}$, Shigeru Matsuoka ${ }^{1,4} \S$, Hiroshi Tsuchikawa ${ }^{1} \S$, Shinya Hanashima ${ }^{1}$, Tohru Oishi $^{1,5}$, Nobuaki Matsumori ${ }^{1,5}$, Wataru Shinoda ${ }^{3,6,7} \dagger$, Michio Murata $^{1,2,4} \dagger$

${ }^{1}$ Department of Chemistry, Graduate School of Science, Osaka University, Toyonaka, Osaka 560-0043, Japan.

${ }^{2}$ Project Research Center for Fundamental Sciences, Osaka University, Toyonaka, Osaka 560-0043, Japan.

${ }^{3}$ Department of Materials Chemistry, Graduate School of Engineering, Nagoya University, Nagoya 464-8603, Japan.

${ }^{4}$ Japan Science and Technology Agency, ERATO, Lipid Active Structure Project, Osaka University, Toyonaka, Osaka 5600043, Japan.

${ }^{5}$ Department of Chemistry, Graduate School of Science, Kyushu University, Fukuoka 819-0395, Japan.

${ }^{6}$ Research Institute for Interdisciplinary Science, Okayama University, Okayama 700-8530, Japan.

${ }^{7}$ Department of Chemistry, Faculty of Science, Okayama University, Okayama 700-8530, Japan.

*These authors contributed equally to this work.

Present addresses are \$Riken, Saitama 351-0198, Japan, and §Oita University, Oita 879-5593, Japan.

$\dagger$ †

\begin{abstract}
:
Amphotericin B, an antifungal drug with a long history of use, forms fungicidal ion-permeable channels across cell membranes. Using solid-state nuclear magnetic resonance spectroscopy and molecular dynamics simulations, we experimentally elucidated the three-dimensional structure of the molecular assemblies formed by this drug in membranes in the presence of fungal sterol, ergosterol. A stable assembly consisting of seven drug molecules was observed to form an ion conductive channel. The structure is somewhat similar to the upper half of the barrel-stave model proposed in the 1970s but substantially different in the number of molecules and in their arrangement. The high-resolution structure explains many previous findings, including structure-activity relationships of the drug, which will be useful for improving drug efficacy and reducing adverse effects.
\end{abstract}


The antifungal antibiotic amphotericin B (AmB, Fig. 1A) is produced by the bacterium Streptomyces nodosus, which was isolated from the soil of the Orinoco River in Venezuela $(1,2)$. AmB has been used for over 60 years to treat systemic fungal infections and remains one of the most clinically important antibiotics (3-5). AmB penetrates the blood-brain barrier and thus serves as a standard therapeutic for most fungal infections of the central nervous system (6). However, serious adverse effects of AmB, such as nephrotoxicity, often cause discontinuation of therapy for life-threatening systemic fungal infections.

The self-assembly of bioactive molecules, such as antimicrobial peptides, in cell membranes has long been a subject of research in structural biology (7). However, despite their pharmacological importance, little is known about the self-assembled structures of nonpeptide natural products such as AmB, mainly due to the lack of a suitable method for structure determination and the difficulties in isotope labeling of the aperiodic and diverse compounds necessary for NMR measurements (8). AmB self-assembles into an oligomeric structure in ergosterol (Erg)-containing membranes of fungal and yeast cells; this oligomer acts as an ion channel and is thought to be responsible for the selective toxicity of the drug against eukaryotic microbes $(4,8,9,10)$. Even now, 50 years after the well-known barrel-stave model (11) was proposed (Fig. 1C), the structure of the AmB channel has not yet been determined. AmB-AmB interactions and functional groups involved in channel formation provide essential information for structural modifications, which are used to improve efficacy and reduce the toxicity of this important antibiotic. Thus, we aimed to determine the entire structure of the AmB channel assembly in Erg-containing membranes and its functionality by the following steps: 1) determine the number of AmB molecules per assembly by solid-state nuclear magnetic resonance (NMR) spectroscopy; 2) determine the interatomic distances between ${ }^{13} \mathrm{C}$-labeled $\mathrm{AmB}$ and ${ }^{19} \mathrm{~F}$ labeled $\mathrm{AmB}$ in the assembly by ${ }^{13} \mathrm{C}-{ }^{19} \mathrm{~F}$ rotational echo double resonance (REDOR) experiments; 3 ) determine how $\mathrm{AmB}$ molecules are arranged in the assembly by using ${ }^{13} \mathrm{C}-{ }^{19} \mathrm{~F}$ distances to yield a timeaveraged structure of the assembly; 4) deduce the structural dynamics of the assembly with molecular dynamics (MD) simulations based on the time-averaged structure; and 5) estimate the ion permeability of the fluctuating assemblies by MD simulations and compare it with previous experimental results. 
A

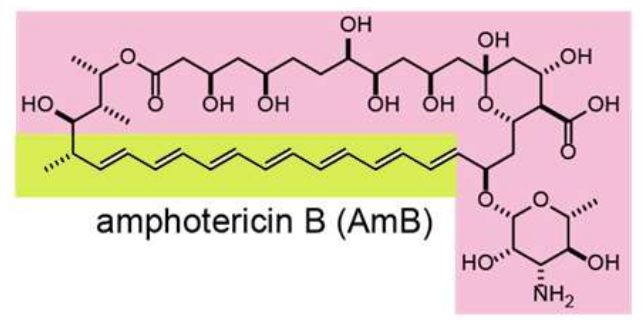

\section{B}

\section{phospholipid}

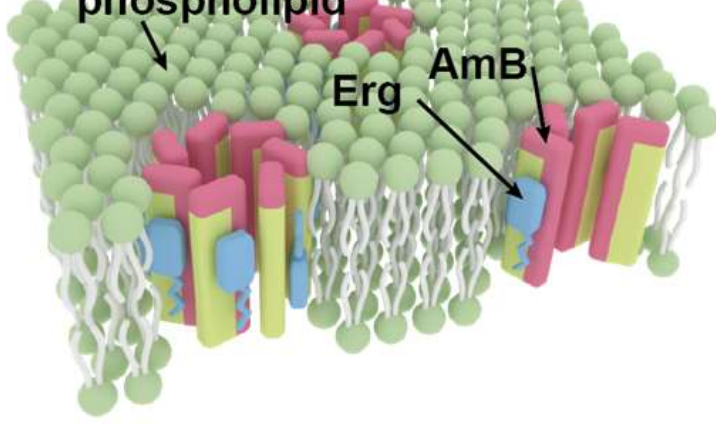

D

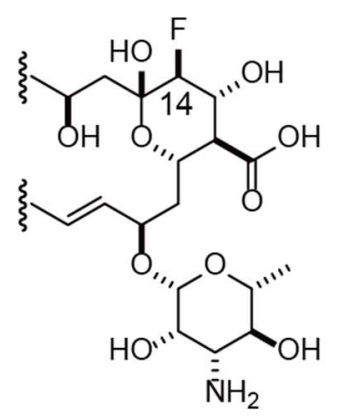

14-F-AmB
E

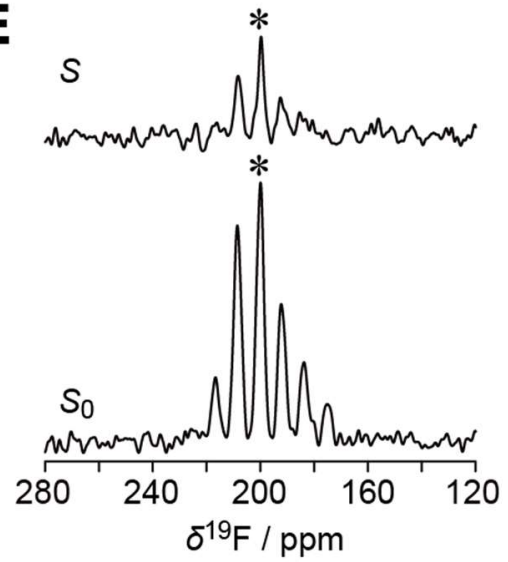

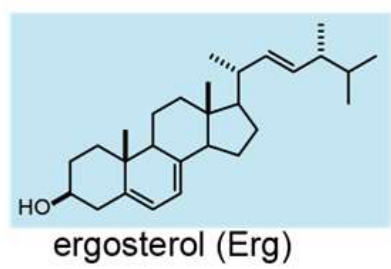

C
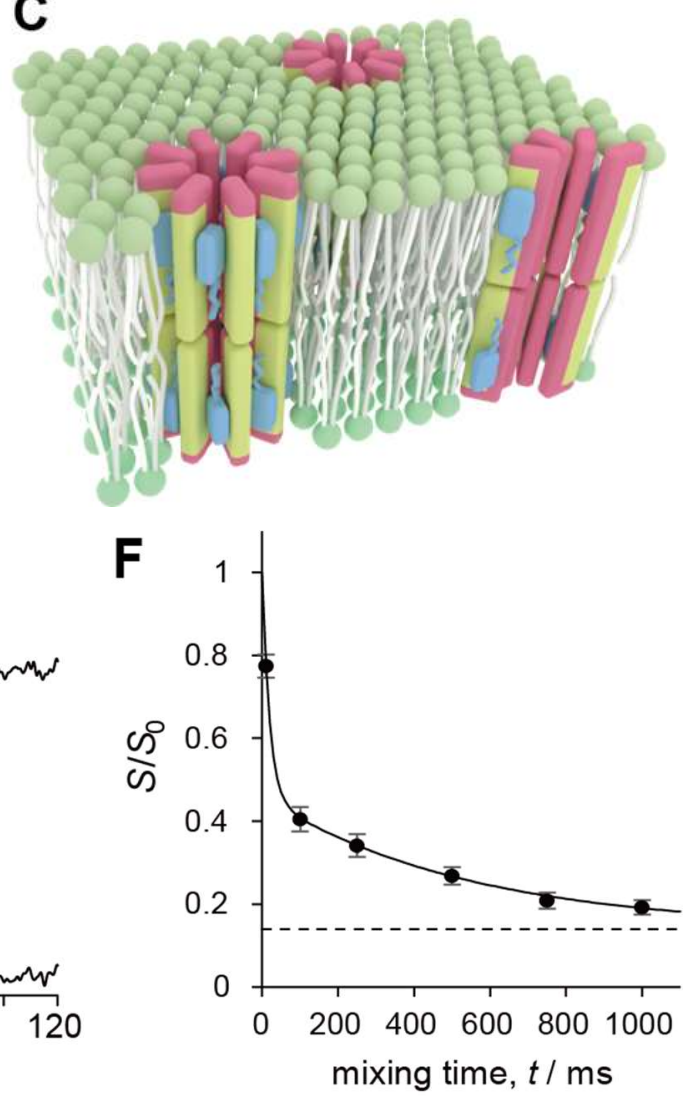

Fig. 1. Structures of AmB ion channel assemblies and the results of a solid-state NMR CODEX method used to determine the number of AmB molecules per assembly. (A) Chemical structures of AmB and Erg. (B) The present model for channel assemblies. (C) The classic barrel-stave model of the AmB ion channel proposed by De Kruijff et al. (11). The hydrophobic and hydrophilic regions of AmB are depicted in greenish yellow and pale magenta, respectively. (D) Partial structure of 14-F-AmB. (E) $S$ (top) and $S_{0}$ (bottom) spectra of a 1:3:7 mixture of 14-F-AmB-Erg-POPC in a ${ }^{19}$ F-CODEX experiment (mixing time: $750 \mathrm{~ms}$ ). The spectra of the sample were obtained at $0^{\circ} \mathrm{C}$ by applying the magic angle spinning method with a rotation speed of $7 \mathrm{kHz}$. The signal in the central band marked by an asterisk (*) corresponds to the signal of AmB that remained outside the membrane (see Fig. S4). (F) The decay curve of $S / S_{0}$, which denotes the fraction of the initial magnetization retained after mixing time. Error bars denote the noise level of the spectra obtained from integration of the baseline near the ${ }^{19} \mathrm{~F}$ signals. The intensity decayed according to the following biexponential equation: $S / S_{0}=0.14+0.54 \mathrm{e}^{(-t / 19.1)}+0.32 \mathrm{e}^{(-t / 541.1)}$. 
This curve asymptotically approaches 0.14 (dotted line), indicating that 14-F-AmB forms a cluster of seven molecules.

\section{How many AmB molecules constitute one channel assembly}

The average number of AmB molecules per channel assembly is crucial information for determining the 3D structure of the AmB channel. In the past, this number has often been estimated by channel conductance measurements $(12,13)$ and MD calculations $(14)$, but the results have been quite widely distributed from four to over ten. Fluorine-labeled AmB (Fig. 1D), which is easily detected in NMR measurements, shows almost the same biological activity as unlabeled AmB (Fig. S1 and Table S1), and a method for its efficient preparation from natural products has been established (15). Using the ${ }^{19} \mathrm{~F}$ centerband-only detection of the exchange (CODEX) method, which is often used to determine the oligomer number of molecules in assemblies $(16,17)$, we attempted to directly determine the number of AmB molecules per channel assembly (Fig. 1E). After removing the influence of unbound AmB (Fig. S4), we measured the $S / S_{0}$ values. These experimental values were plotted as a function of mixing time and found to lie on a curve that asymptotically approached 0.14 , which is the reciprocal of seven (Fig. 1F). These results implied that the average number of $\mathrm{AmB}$ molecules per assembly is seven because the magnetization of one AmB molecule diffuses equally toward the other neighboring molecules in the assembly during a long mixing time. However, the results do not rule of the out the possibility that assemblies with six and eight molecules coexist within the membrane.

\section{Structural overview of AmB assembly in membranes}

We next determined the interatomic distances between adjacent AmB molecules in the assembly using solid-state NMR (Fig. 2). Skipped ${ }^{13} \mathrm{C}$-labeled AmB was obtained by a biosynthetic method (Fig. 2C). AmBs fluorinated at positions 14 or 32 and $26,40-{ }^{13} \mathrm{C}_{2}$-AmB (Fig. 2C) were prepared by chemical synthesis $(15,18)$ (Supplementary Materials and Methods). Two lipid bilayer models with different lipid compositions and different Erg concentrations in palmitoyloleoylphosphatidylcholine (POPC) were used for ${ }^{13} \mathrm{C}-{ }^{19} \mathrm{~F}$ rotational echo double resonance (REDOR, Fig. 2A) experiments because we used a low concentration of Erg to measure REDOR for $26,40-{ }^{13} \mathrm{C}_{2}$-AmB due to signal overlap with Erg (Figs. 2A and S2). Six parameters (Figs. 3A, B and Figs. S3, S5) had to be determined to elucidate the 3D structure of the AmB channel assembly: the number of molecules $n$ per assembly, the Euler angles $(\alpha, \beta, \gamma)$, the channel inner diameter $R$, and the adjacent probability $p$, as described in Fig. S5. Therefore, it was necessary to measure the distance by using as many labeled atom pairs as possible; we adopted five REDOR-derived distances (Fig. 2B) plus the CODEX result for determining the parameters. For the REDOR experiment, time-dependent increases in the $\Delta S / S_{0}$ values were observed for each ${ }^{13} \mathrm{C}-{ }^{19} \mathrm{~F}$ pair (Fig. 2B), indicating that 
molecular motion was slow enough to allow accurate measurements of the ${ }^{13} \mathrm{C}-{ }^{19} \mathrm{~F}$ distances in the membrane. With these distance constraints in hand, we investigated an assembly structure that could account for the NMR results without being constrained by previous models, such as the barrel-stave model and sterol-sponge model (19). Recently, we found that the vertical penetration of AmB through the lipid bilayer corresponds to the length of a single drug molecule under experimental conditions similar to those used in this study $(20,21)$. The present REDOR experiment showed that the AmB molecules in the assembly are arranged in a parallel manner, as assumed in the classic barrel-stave model (Fig. S6). Taken together, the results strongly suggest that the AmB assembly resembles the upper half of the barrel-stave model (Figs. 1B and S7), and other possible structures, such as the sterol-sponge model, are considered extremely unlikely.
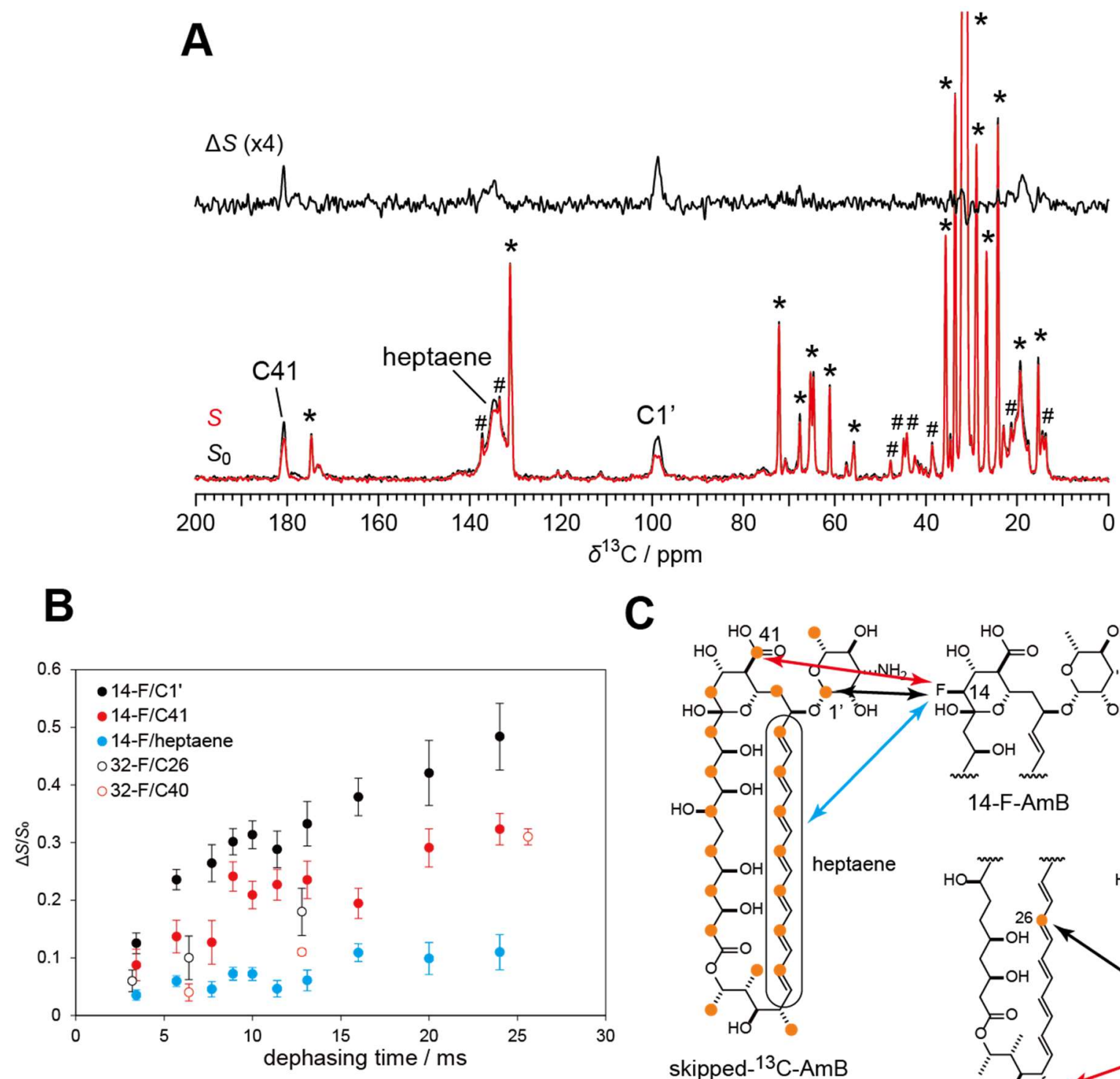

C

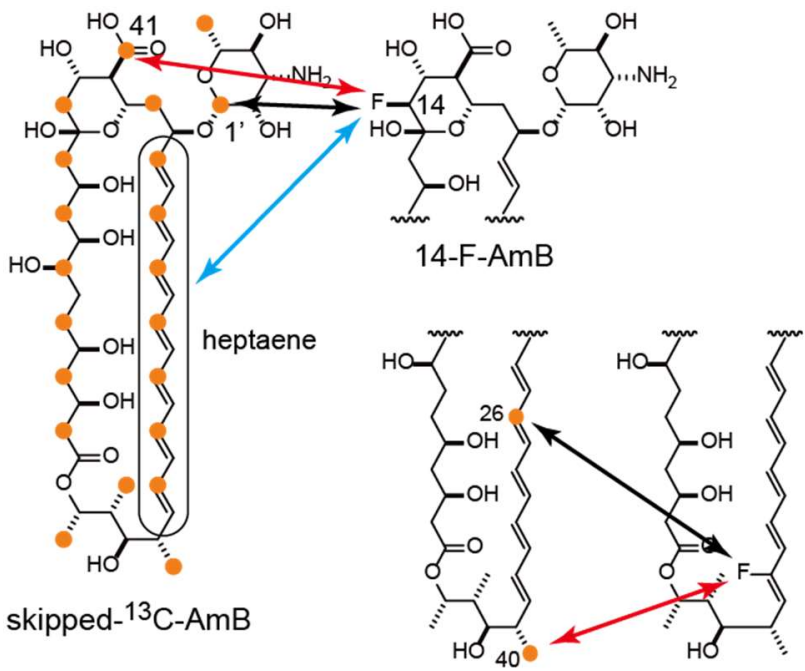

$26,40-{ }^{13} \mathrm{C}_{2}-\mathrm{AmB}$

32-F-AmB

Fig. 2. Solid-state NMR experiments used to determine intermolecular ${ }^{13} \mathrm{C}-{ }^{19} \mathrm{~F}$ distances. 
(A) ${ }^{13} \mathrm{C}\left\{{ }^{19} \mathrm{~F}\right\}$ REDOR spectra of $14-\mathrm{F}-\mathrm{AmB} /$ skipped- ${ }^{13} \mathrm{C}-\mathrm{AmB} / \mathrm{Erg} / \mathrm{POPC}=0.5 / 0.5 / 3 / 7$ hydrated with 10 mM HEPES containing $\mathrm{D}_{2} \mathrm{O}$ at $25^{\circ} \mathrm{C}$. The sample spinning speed was $7 \mathrm{kHz}$, and the dephasing time was $16 \mathrm{~ms}$. The full echo ( $S_{0}$, bottom, black), REDOR-dephased ( $S$, bottom, red), and difference ( $\Delta S$, top) spectra clearly showed a reduction in the peak intensity of the $\mathrm{C} 41$, heptane and $\mathrm{C} 1$ ' signals; * and ${ }^{\#}$ indicate the signals attributed to POPC and Erg, respectively. (B) REDOR dephasing observed for C1' (filled black), C41 (filled red), and heptaene (filled blue) upon irradiation at 14-F and for C26 (open black) and $\mathrm{C} 40$ (open red) upon irradiation at 32-F. The error bars denote the $\mathrm{S} / \mathrm{N}$ ratios of $\Delta S$ signals. $(\mathbf{C}){ }^{13} \mathrm{C}-$ labeled positions of the skipped labeled and synthetic AmBs shown with orange circles. Arrows denote the interatomic distances determined by REDOR and are colored in the same manner as the dots in (B).

\section{Time averaged structure of AmB assembly derived from NMR}

We generated a time-averaged structure of the AmB assembly as follows: the upper half of the barrelstave model was used as a starting point for an AmB self-assembled structure (Table S2) (22)(23). We varied the six parameters that defined the structure to minimize the root mean square deviation (RMSD) between the interatomic distances in the assembly model and the REDOR-derived distances (Figs. S5, S8). Fig. $3 \mathrm{C}$ shows curves of the experimental $\Delta S / S_{0}$ values obtained for each interatomic distance in the AmB assembly as a function of dephasing time. For the interatomic 14-F/C41, 14-F/C1' and 32-F/C40 distances, two distances, $r_{1}$ and $r_{2}$ (Fig. 3B), were obtained for each labeled carbon atom; the 32-F/C26 distance deviated slightly from the measured value, presumably because of an interaction between assemblies (Fig. $\mathrm{S} 15)$. This is the first experimentally validated structure of the AmB assembly in a membrane (Fig. 3D). In the structure, the molecular axis of AmB is nearly perpendicular to the central axis (the cone angle $\beta$ is approximately $2^{\circ}$ ), and the channel radius at the oxygen atom at $\mathrm{C} 8$ is $5.4 \AA$. 

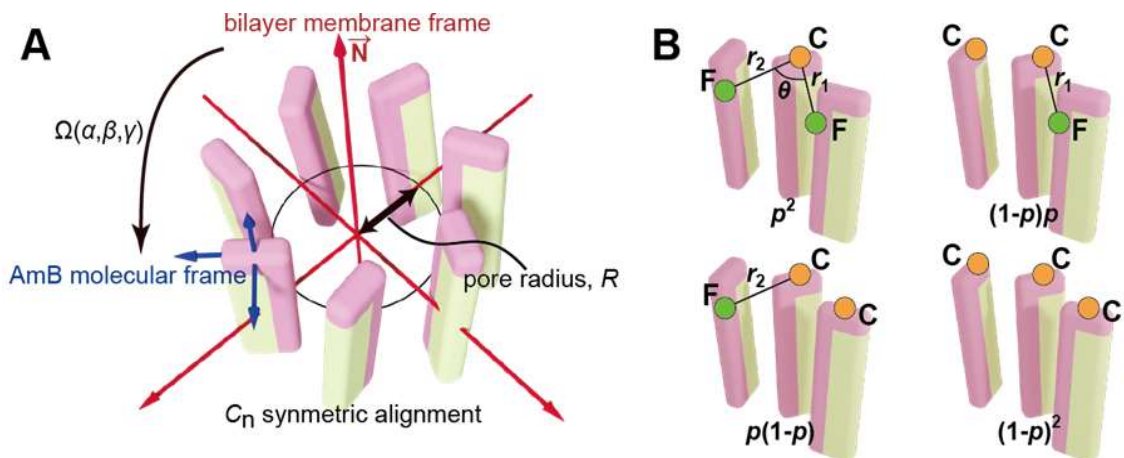

C
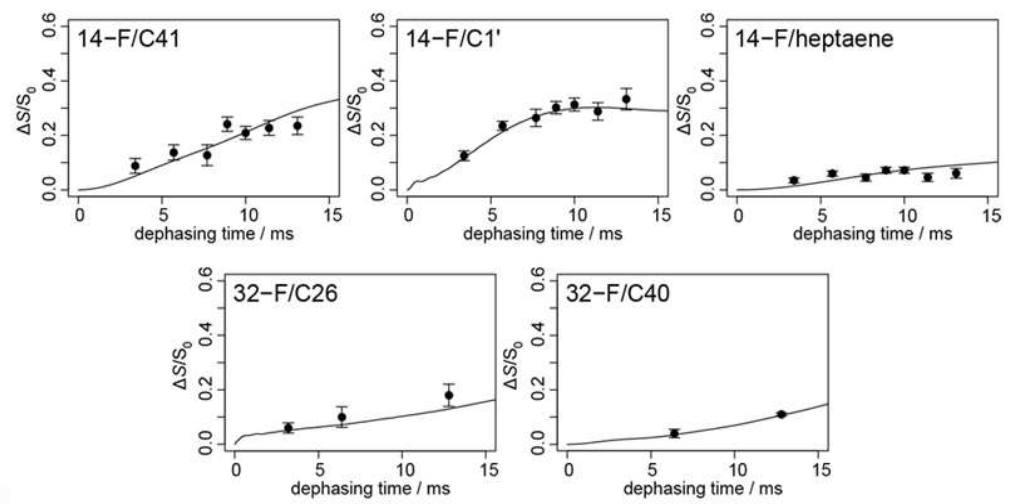

D

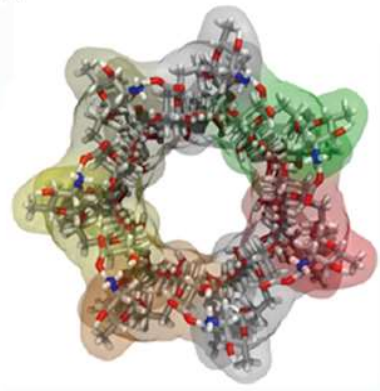

E

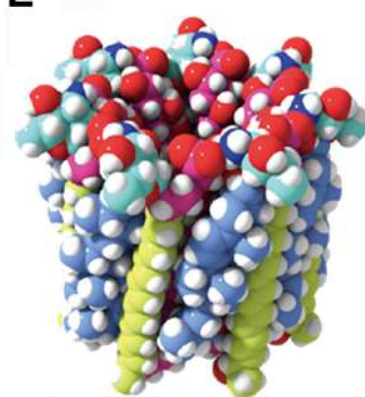

Fig. 3. Construction of a symmetric structure of the AmB assembly based on the interatomic distances obtained via NMR. (A) Definition of the parameters $\alpha, \beta, \gamma, n$, and $R$ (see Fig. S5). (B) Possible adjacent combinations of ${ }^{19} \mathrm{~F}-\mathrm{AmB}$ and ${ }^{13} \mathrm{C}-\mathrm{AmB}$ and the abundance ratios for the possibility parameter $p$ (Fig. S3). (C) Simulated REDOR dephasing curves calculated from the best fitted parameters $(\alpha, \beta, \gamma, n, R$, and $p)=\left(-12^{\circ}, 2^{\circ},-20^{\circ}, 7,5.4 \AA\right.$, and 0.24$)$ and experimental dephasing values (same as Fig. 2B). (D) Top and side views of the symmetric structure of the heptamer (seven-molecule) assembly. (E) Space-filling model of the seven AmB and seven Erg complex.

\section{Dynamic structure of AmB assembly deduced by MD simulation}

To clarify the structure of a functional ion channel from the AmB-AmB configuration derived from NMR, we next simulated the molecular dynamics of the AmB assembly using MD calculations. The timescale of the REDOR experiment is in milliseconds, which is much slower than the molecular fluctuations occurring in the submicrosecond range in lipid bilayers. Therefore, the magnitude of REDOR 
dephasing reflects the time-averaged ${ }^{13} \mathrm{C}-{ }^{19} \mathrm{~F}$ distances in the AmB assembly, and these distances correspond to a static structure with centrosymmetry (Fig. 3D, E). It is important to know how the channel structure varies with time to more fully understand the molecular mechanisms of ion flux, which is responsible for the pharmacological activities of AmB. An accurate initial structure (Fig. 4A) is essential for simulating a dynamic structure because the simulation time is limited to microseconds with even the fastest computers, but the exchange of interacting molecules in the assembly occurs much more slowly than that in the simulation. In other words, the following events cannot occur during the simulation time: a change in the number of AmB molecules per assembly, an exchange between the single length assembly (Fig. 1A) and the double length assembly (Fig. 1B), or flipping of the AmB or Erg structure. Fig. 4B and 4C show a typical snapshot and the average structure estimated from MD simulations, respectively. Each Erg molecule generally continues to interact with the same AmB molecule during the $1 \mu$ s simulation (Figs. 4B, 4D and S11). These results are consistent with a previous report that the AmB-Erg complex has a lifetime longer than the NMR timescale (18). In addition, the averaged interatomic distances between isotope-labeled positions were calculated to directly compare the channel structures obtained from solidstate NMR to those obtained by MD simulations, and the results were in good agreement (Fig. 4E and Table S3).

\section{Ion conducting activity of AmB assembly evaluated by MD simulation}

To evaluate how well the structure of the AmB assembly constructed on the basis of NMR and MD results emulates the real ion channel responsible for drug activity, we measured the conductance of the assembly under conditions similar to those used for single channel-recording experiments (24). The channel activity of the dynamic AmB assembly was estimated in Erg-containing diphytanoylphosphatidylcholine (DPhPC) membranes with MD simulations (Figs. S13, S21-S24 and Table S6-S8). As shown in Fig. 5A, 5B, the heptamer channel best reproduced the experimental ion permeability (24), and the formation of a water column in the channel pore was deduced to significantly stabilize the channel structure (Fig. 4C). Assemblies consisting of six or fewer AmB molecules did not allow $\mathrm{K}^{+}$passage, whereas assemblies of eight molecules allowed much more $\mathrm{K}^{+}$to pass through than was observed experimentally (Fig. 5A). 


\section{Discussion}

In this study, we investigated the ion channel structure of AmB via a new approach involving solidstate NMR spectroscopy and MD simulations. Although the resulting channel structure shares similarities with the classic structure (11) proposed in the 1970s, significant differences were observed: the number of $\mathrm{AmB}$ molecules per conductive ion channel is seven rather than eight, the macrocycle plane is arranged not radially but in a spiral configuration (Fig. 3D, 3E), and the length of the conductive AmB channel that penetrates the lipid bilayer corresponds to the length of a single molecule rather than the length of two molecules (Fig. 1B/C, and Fig. S12), even though the single length assembly was assumed to be too short to function as an ion channel (14). We also found that intermolecular hydrogen bonds from the amino sugar moieties in AmB-AmB and AmB-Erg provide an important force that stabilizes these ion channels in an active form (Fig. 4D); the AmB-AmB contact is further stabilized by a salt bridge between the carboxyl group of $\mathrm{C} 41$ and the amino group at $\mathrm{C} 3$ '. In addition, the hydrogen bond formed between the $\mathrm{C} 2$ '-OH of one $\mathrm{AmB}$ and the $\mathrm{C} 15-\mathrm{OH}$ of an adjacent AmB stabilizes the channel complex (Table S5).

We have to consider how similar the present AmB channel formed in the model bilayers is to the real ion channels that exert antifungal activity in the human body. The apparent difference in the conditions of channel formation involves the concentration of $\mathrm{AmB}$ in the membranes since the $\mathrm{AmB}$ concentration for NMR measurements was approximately 100 times that found in real fungal membranes (5). First, we investigated with UV-VIS and CD spectroscopy to determine whether the 3D structure of the channel assembly changed with changes in the concentration of AmB in the membrane. The UV-VIS spectra (Fig. S9) revealed that the heptaene moiety of AmB in the Erg-containing membrane showed typical absorbance bands with sharper and redshifted peaks, which were distinct from those of Cho-containing or sterol-free membranes. These features were unaffected by the concentration of $\mathrm{AmB}$, indicating that the heptaene moiety resided stably in the membrane interior at concentrations ranging from $0.1 \mathrm{~mol} \%$ to $10 \mathrm{~mol} \%$ of those of total lipids (25). The CD spectra of AmB, which sensitively reflect the molecular arrangements of $\mathrm{AmB}$ in membranes (26), showed that AmB formed essentially the same assembly over the same concentration range used for UV-VIS measurements (Fig. S10). Therefore, we presume that the structure of AmB assemblies elucidated by solid-state NMR is basically the same as that of ion channels occurring in fungal membranes at low concentrations of the drug. Usually, peptides remain in a monomeric form at very low membrane concentrations and tend to form assemblies with a larger number of molecules at higher concentrations (27). Why do AmB molecules form a similar assembly despite the large concentration difference? We speculate that this similarity can be explained by the stability of the heptamer assembly (Fig. S8) and the resulting aggregation of the channel assemblies. As shown in Figs. 5C and 5D 
(also Figs. S15, S25, and S26), the number of unstable interdigitating lipids surrounding the assemblies is expected to be reduced by aggregation of the channels. Even if the overall concentration of AmB is low, as in the case of antifungal assays, the AmB concentration is assumed to increase locally as a result of aggregation of the assemblies.

Accurately elucidating the channel structure enables us to explain some previously reported structureactivity relationships. For example, the destructive effect of $\mathrm{N}$-acylation on the antifungal activity of AmB and the retained activity of $\mathrm{AmB}$ methyl ester (AME), which was subjected to human clinical trials, were demonstrated by MD simulations (28) (Figs. S14, S27 and Tables S9, S10); the simulated channel activity of AME also reproduced previous experimental values (29) (Fig. 5A). In mammalian cell membranes containing Cho, the interaction between $\mathrm{AmB}$ and $\mathrm{Cho}$ is too weak to form stable ion channels or the resulting channel aggregates (Figs. S11, S20), often leading to unstructured amorphous of AmB outside membranes, as revealed by the UV-VIS spectra (20) (Figs. S9, S16). Nevertheless, AmB channels occurring in mammalian cells could be very similar in structure to those in Erg-containing membranes (Fig. S10G and Table S5) and thus weakly increase the ion permeability of the membrane by allowing a small number of ions to pass, which results in the adverse effects of the drug.

We elucidated the dynamic structure of the assembly using NMR experiments and MD simulations. To investigate whether this molecular assembly functions as an ion channel, the value for conductance of $\mathrm{K}^{+}$ obtained by MD simulation was compared with the experimental values from single channel recording. In fact, the two values agreed well, and it is suggested that the structure of the AmB channel determined in this study is more accurate in terms of the number of molecules per channel and the mode of AmB-AmB interaction than those previously proposed. The present 3D structure of the AmB channel could, therefore, be useful for improving drug efficacy and reducing adverse effects. 


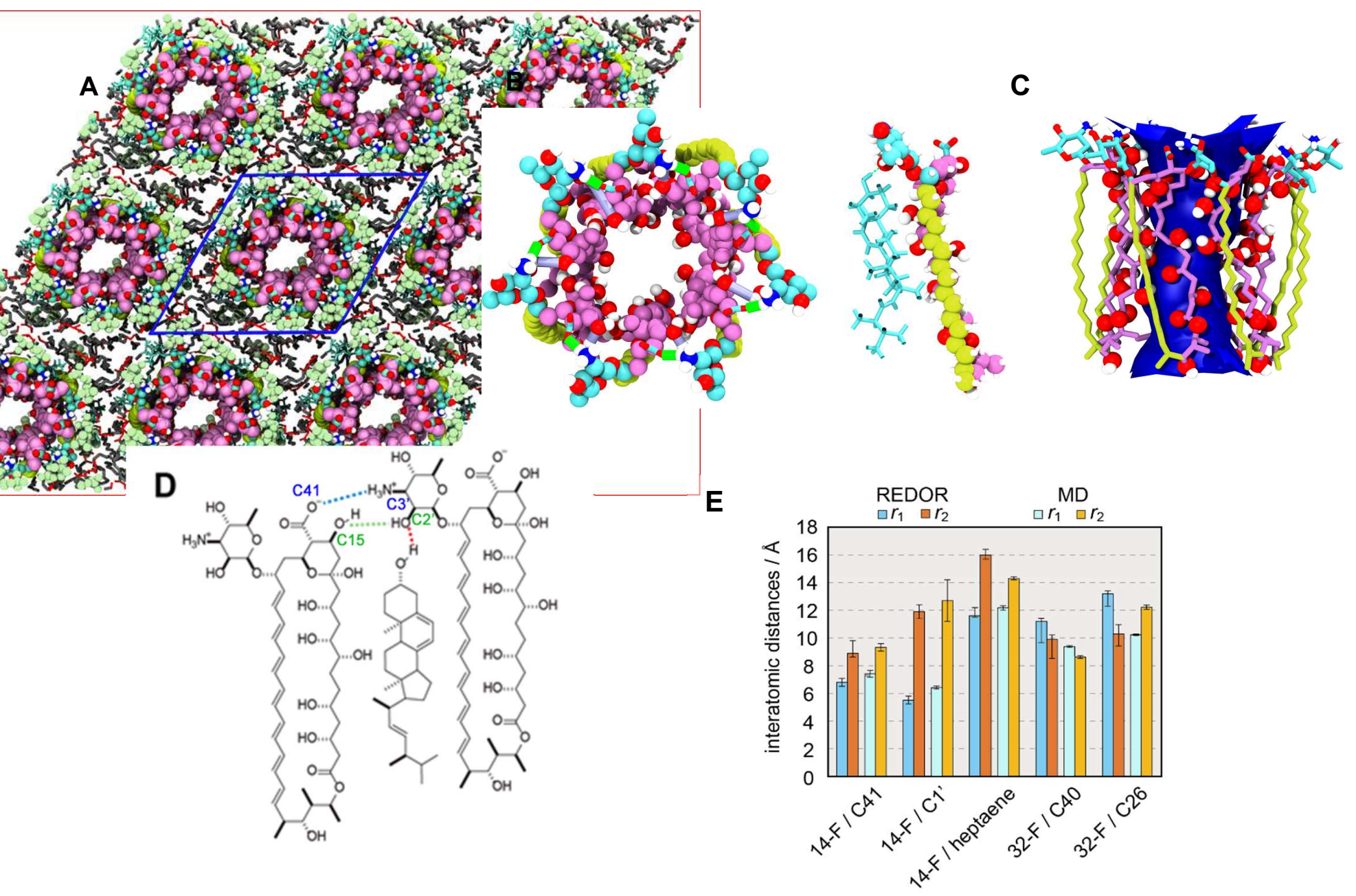

Fig. 4. Construction of asymmetric assembly based on MD simulations. (A) Initial structure of the AmB assemblies with periodic boundary conditions constructed on the basis of NMR results. (B) Snapshots of allatom MD simulations of an AmB assembly and an AmB-Erg complex. (C) Average structure of an AmB assembly showing the water-accessible region inside the pore. (D) Major intermolecular interactions stabilizing the AmB-Erg channel assembly. (E) Interatomic distances measured by solid-state NMR experiments and the values from MD simulations. The distances differ between the front and back of the molecule and correspond to $r 1$ and $r 2$ (Fig. 3B); the structure obtained by MD is not rotationally symmetric, so the average $\pm \mathrm{SD}$ are shown, where SD obtained from ten runs of the simulation were less than 5\%. 
A

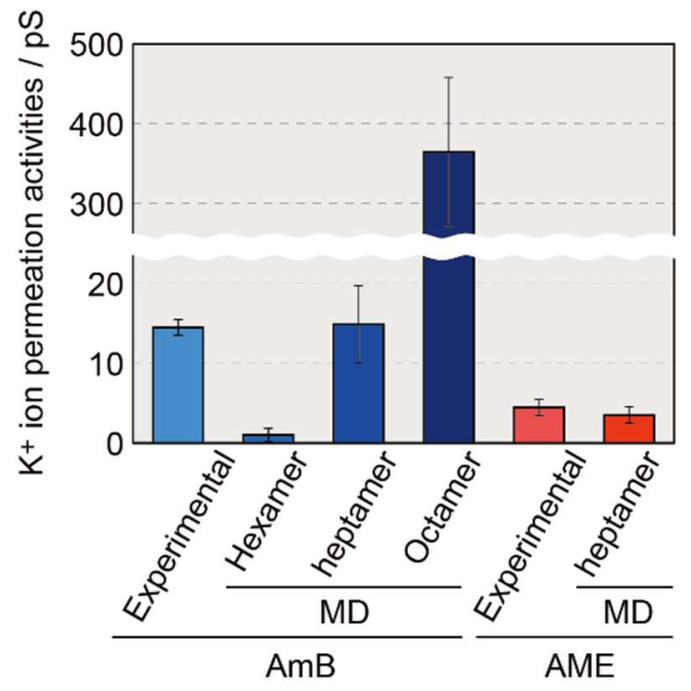

C
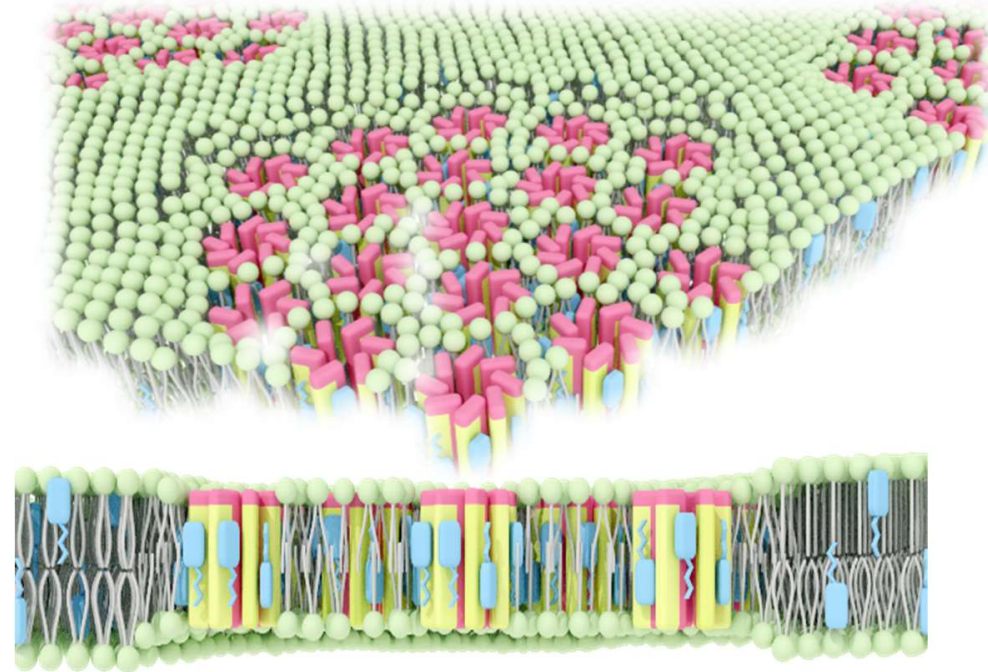

B
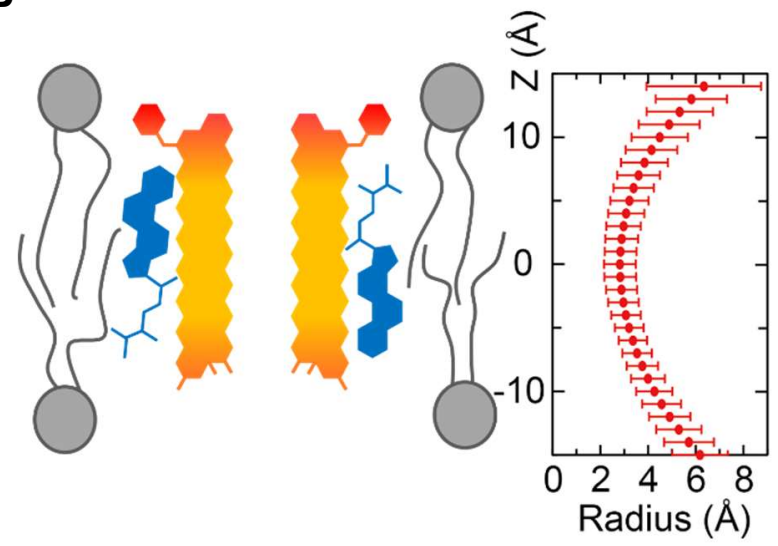

D

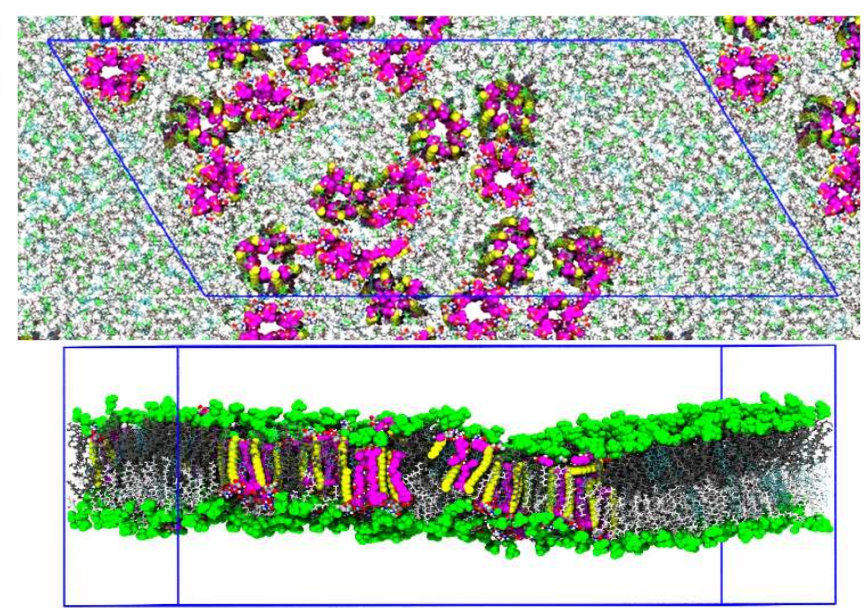

Fig. 5. Ion channel activity of AmB assembly based on MD simulations and aggregate formation of AmB assemblies in membrane. (A) Experimental and MD-derived values of $\mathrm{K}^{+}$permeability $(24,29)$; the experiments in the literature carried out by using the membranes of DPhPC-Erg 5:1 (2 M KCl, at $100 \mathrm{mV})$ (24) and egg yolk lecithin (29). This clearly indicates that the channel that shows a value close to those of past single channel measurements is the heptamer assembly. (B) Channel radius profile (distance from the center of a water molecule) along the horizontal axis obtained for the present channel structure. The horizontal bars denote the standard deviation of the channel radius during the simulation. (C) Illustration of an aggregate of channel assemblies formed in an artificial membrane, where tens of assemblies are aggregated with lipid interdigitations (see Fig. S16 for MD simulation). (D) The snapshot of the partial aggregation of AmB-Erg assemblies after $2 \mu$ s MD simulation under the similar conditions to those of NMR experiments with the antiparallel channel-channel arrangement. The blue frame shows the periodic boundary box (also see Fig. S26). 


\section{New References and Notes:}

1. R. Donovick, W. Gold, J. F. Pagano, H. A. Stout, Amphotericins A and B, antifungal antibiotics produced by a streptomycete. I. In vitro studies. Antibiotics Annual, 3, 579-586 (1956)

2. E. T. Stiller, J. Vandeputte, J. L. Wachtel, Amphotericins A and B, antifungal antibiotics produced by a streptomycete. II. The isolation and properties of the crystalline amphotericins. Antibiotics Annual. 3, 587-591(1956).

3. S. Hartsel, J. Bolard, Amphotericin B: new life for an old drug. Trends Pharmacol. Sci. 17, 445-449 (1996).

4. J. Bolard, How do the polyene macrolide antibiotics affect the cellular membrane properties? Biochim. Biophys. Acta 864, 257-304 (1986).

5. D. Ellis, Amphotericin B: spectrum and resistance. J. Antimicrob. Chemother. 49, 7-10 (2002).

6. V. Petraitis, R. Petraitiene, J. M. Valdez, V. Pyrgos, M. J. Lizak, B. A. Klaunberg, D. Kalasauskas, A. Basevicius, J. D. Bacher, D. K. Benjamin Jr., W. W. Hope, T. J. Walsh. Amphotericin B penetrates into the central nervous system through focal disruption of the blood-brain barrier in experimental hematogenous Candida meningoencephalitis, Antimicrob. Agents Chemother. 63, e01626-19 (2019).

7. W. Luo, M. Hong, Determination of the oligomeric number and intermolecular distances of membrane protein assemblies by anisotropic ${ }^{1} \mathrm{H}$-driven spin diffusion NMR spectroscopy. J. Am. Chem. Soc. 128, 7242-7251 (2006).

8. A. Vertut-Croquin, J. Bolard, C. M. Gary-Bobo, Enhancement of amphotericin B selectivity by antibiotic incorporation into gel state vesicles. A circular dichroism and permeability study. Biochem. Biophys. Res. Commun. 125, 360-366 (1984).

9. S. A. Davis, B. M. Vincent, M. M. Endo, L. Whitesell, K. Marchillo, D. R. Andes, S. Lindquist, M. D. Burke, Nontoxic antimicrobials that evade drug resistance. Nat. Chem. Biol. 11, 481-487 (2015).

10. P. Dong, C. Zong, Z. Dagher, J. Hui, J. Li, Y. Zhan, Polarization-sensitive stimulated Raman scattering imaging resolves amphotericin B orientation in Candida membrane, Sci. Adv. 7, eabd5230 (2021).

11. B. de Kruijff, R. A. Demel, Polyene antibiotic-sterol interactions in membranes of Acholeplasma laidlawii cells and lecithin liposomes. 3. Molecular structure of the polyene antibiotic-cholesterol complexes. Biochim. Biophys. Acta 339, 5770 (1974).

12. B. Venegas, J. González-Damián, H. Celis, I. Ortega-Blake, Amphotericin B channels in the bacterial membrane: role of sterol and temperature. Biophys. J. 85, 2323-2332 (2003).

13. J. Gabrielska, M. Gagoś, J. Gubernator, W. I. Gruszecki, Binding of antibiotic amphotericin B to lipid membranes: A ${ }^{1} \mathrm{H}$ NMR study. FEBS Lett. 580, 2677-2685 (2006).

14. J. Zielińska, M. Wieczor, P. Chodnicki, E. Grela, R. Luchowski, Ł. Nierzwicki, T. Bączek, W. Gruszecki, J. Czub, Selfassembly, stability and conductance of amphotericin B channels: bridging the gap between structure and function. Nanoscale 13, 3686-3697 (2021).

15. N. Matsumori, Y. Umegawa, T. Oishi, M. Murata, Bioactive fluorinated derivative of amphotericin B. Bioorganic Med. Chem. Lett. 15, 3565-3567 (2005).

16. E. R. deAzevedo, W.-G. Hu, T. J. Bonagamba, K. Schmidt-Rohr, Centerband-only detection of exchange: Efficient analysis of dynamics in solids by NMR. J. Am. Chem. Soc. 121, 8411-8412 (1999).

17. R. Mani, S. D. Cady, M. Tang, A. J. Waring, R. I. Lehrer, M. Hong, Membrane-dependent oligomeric structure and pore formation of a $\beta$-hairpin antimicrobial peptide in lipid bilayers from solid-state NMR. Proc. Natl. Acad. Sci. USA 103, 16242-16247 (2006).

18. Y. Nakagawa, Y. Umegawa, N. Matsushita, T. Yamamoto, H. Tsuchikawa, S. Hanashima, T. Oishi, N. Matsumori, M. Murata, The structure of the bimolecular complex between amphotericin B and ergosterol in membranes is stabilized by face-to-face van der Waals interaction with their rigid cyclic cores. Biochemistry 55, 3392-3402 (2016).

19. T. M. Anderson, M. C. Clay, A. G. Cioffi, K. A. Diaz, G. S. Hisao, M. D. Tuttle, A. J. Nieuwkoop, G. Comellas, N. Maryum, S. Wang, B. E. Uno, E. L. Wildeman, T. Gonen, C. M. Rienstra, M. D. Burke, Amphotericin forms an extramembranous and fungicidal sterol sponge. Nat. Chem.Biol. 10, 400-406 (2014).

20. T. Yamamoto, Y. Umegawa, H. Tsuchikawa, S. Hanashima, N. Matsumori, K. Funahashi, S. Seo, W. Shinoda, M. Murata, The Amphotericin B-ergosterol complex spans a lipid bilayer as a single-length assembly. Biochemistry 58, 5188-5196 (2019). 
21. T. Yamamoto, Y. Umegawa, M. Yamagami, T. Suzuki, H. Tsuchikawa, S. Hanashima, N. Matsumori, M. Murata, The perpendicular orientation of amphotericin B methyl ester in hydrated lipid bilayers supports the barrel-stave model. Biochemistry 58, 2282-2291 (2019).

22. K. N. Jarzembska, D. Kamiński, A. A. Hoser, M. Malińska, B. Senczyna, K. Woźniak, M. Gagoś, Controlled crystallization, structure, and molecular properties of iodoacetylamphotericin B. Cryst. Growth Des. 12, 2336-2345 (2012).

23. N. Matsumori, Y. Sawada, M. Murata, Mycosamine orientation of amphotericin B controlling interaction with ergosterol: Sterol-dependent activity of conformation-restricted derivatives with an amino-carbonyl bridge. J. Am. Chem. Soc. 127, 10667-10675 (2005).

24. R. A. Brutyan, P. McPhie, On the one-sided action of amphotericin B on lipid bilayer membranes. J. Gen. Phisiol. 107, 6978 (1996).

25. G. Fujii, J. E. Chang, T. Coley, B. Steere, The formation of amphotericin B ion channels in lipid bilayers. Biochemistry 36, 4959-4968 (1997).

26. A. Vertut-Croquin, J. Bolard, M. Chabbert, C. Gary-Bobo, Differences in the interaction of the polyene antibiotic amphotericin B with cholesterol- or ergosterol-containing phospholipid vesicles. A circular dichroism and permeability study. Biochemistry 22, 2939-2944 (1983).

27. H. W. Huang, Molecular mechanism of antimicrobial peptides: The origin of cooperativity. Biochim. Biophys. Acta 1758, 1292-1302 (2006).

28. M. Chéron, B. Cybulska, J. Mazerski, J. Grzybowska, A. CzerwiŃski, E. Borowski, Quantitative structure-activity relationships in amphotericin B derivatives. Biochem. Pharmacoly. 37, 827-836 (1988).

29. S. C. Hartsel, S. K. Benz, W. Ayenew, J. Bolard, $\mathrm{Na}^{+}, \mathrm{K}^{+}$and $\mathrm{Cl}^{-}$selectivity of the permeability pathways induced through sterol-containing membrane vesicles by amphotericin B and other polyene antibiotics. Eur. Biophys. J. 23, 125-132 (1994).

Acknowledgments: Funding: The calculations were performed using the facilities of the supercomputer center at the Research Center for Computational Science (Okazaki, Japan) and the Institute for Solid State Physics, The University of Tokyo (Tokyo, Japan).

This work was supported by JSPS KAKENHI Grant Numbers $16 \mathrm{H} 06315$ to U. Y., W. S., and M.M.; and 17H06406 to H. T. and M.M. and by JST ERATO Lipid active structure project (JPMJER1005).

Competing interests: The authors declare no competing interests.

Data and materials availability: All data are available in the manuscript or the supplementary materials.

\section{SUPPLEMENTARY MATERIALS}

Materials and Methods with Figs. M1-M17 and Tables M1-M2 including the spectra of synthetic materials of isotope-labeled AmB.

Supplementary figures (Figs. S1-S27) and tables (Tables S1-S10) with text 